\section{PROTEINS}

\section{Enzymes Releused}

from our Molecular Biology Correspondent

A LARGE number of the enzymes of the greatest interest are confined, like a genie in a bottle, to the interior of a cell membrane, and often expire when they are liberated. An increasing number are now coming to light, however, which are active in aqueous media, or sometimes in simulated solvent conditions which approach more or less those of the membrane. The properties of some of these proteins are engrossing: a recent example is the enzyme $\mathrm{C}_{55}$-isoprenoid alcohol phosphokinase (Sandermann and Strominger, Proc. US Nat. Acad. Sci., 68, 2441; 1971) which has been found to contain no less than some 60 per cent of non-polar amino-acids, and partitions out of water into wet butanol.

Another enzyme, and one that should commend itself to anybody looking for a ride on the gravy-train of socioeconomic relevance, is the DDTase that occurs in resistant strains of houseflies. Because the solubility of DDT in water is of the order of $10^{-9}$ moles $/ 1$., it must be assumed that the DDT does its work in a lipid milieu, and that this too is where the enzyme is to be found. The location of the DDTase, or more precisely DDT-dehydrochlorinase, has not in fact been determined, but a new study by Dinamarca, Levenbook and Valdés (Arch. Biochem., 147, 374; 1971) has now established that it is a lipoprotein: a considerable quantity of phospholipid is associated with it, and it has not proved possible to extract it without denaturation.

The enzyme has a number of curious features. In the presence of a reducing agent, such as mercaptoethanol, it exists as a monomer of 30,000 molecular weight, but as prepared in the absence of thiols, it is an enzymatically active tetramer. Moreover, addition of substrate to the monomer causes it to associate into tetramers, which appear to be the only active species. At low concentrations of reducing agent the dimer and trimer can also be observed in gel electrophoresis. The protein has a high thiol content, and no disulphide bonds can be detected, even in the tetramer, which leaves the effect of the reducing agent unexplained and seemingly inexplicable. The paradox is compounded by the observation that reduced glutathione, actually a cofactor in the catalytic process, stabilizes the tetrameric form. Taking the results at face value, one can only speculate rather helplessly about thiol groups of very low redox potential.

The four subunits are probably identical, for they are homogeneous in gel electrophoresis, produce the right number of tryptic peptides for the content of lysine and arginine calculated on a monomer basis, and have only one kind each of $\mathrm{C}$-terminal and $\mathrm{N}$-terminal amino-acids. DDTase is a thiol enzyme in the sense that titration with a mercurial leads to progressive inactivation. In the absence of substrate some of the thiols react quickly, others more slowly, presumably with unfolding, but when DDT is present all of them become unreactive. Dinamarca et al. suggest that the phospholipid associated with the enzyme has the function of dissolving the DDT, and presumably leading it to the active site. The alternative of course is that it comes from a membrane in which the enzyme resides.

A membrane protein which has attracted the attention of Kornberg's laboratory is the phospholipase A of Escherichia coli (Scandella and Kornberg, Biochemistry, 10, 4447; 1971). This turned up in healthy cells, which were being screened as a control for phage-infected cells, on the principle that the latter might be expected to have a specific phage-induced phospholipase. Such an enzyme could open a channel in the membrane, through which the products of the phage genome, such as lysozyme, would pass. The $E$. coli enzyme is evidently embedded in the membrane, for it is released only by procedures that break down the membrane structure, and it is not susceptible in situ to proteolytic attack. Its specificity is unusual in that it will act on
L-phosphatidylcholine, but not on the D-enantiomer. As isolated, the phospholipase contains lipid, but this may be solvent-extracted without detriment to the enzymatic activity. The lipidfree protein, however, forms aggregates.

Among the more diverting properties of this enzyme is its ability to survive exposure to concentrated sodium dodecyl sulphate. Whether this is a matter of renaturation after removal of the detergent or of persistence of the native conformation in its presence is uncertain, however, for the alkyl sulphate is a competitive inhibitor and the assays cannot be performed in its presence. This is a finding which should persuade workers in the membrane field to moderate their faith in the infallible dissociating and denaturing capacity of the dodecyl sulphate ion.

Another type of membrane protein, evidently not an enzyme, but serving a vital function nonetheless, has been isolated from $E$. coli by Weiner and Heppel (J. Biol. Chem., 246, 6933; 1971). It binds glutamine with an equilibrium constant of $10^{-7} \mathrm{M}^{-1}$, and is presumed to be involved in the active transport of this amino-acid. The protein is released from the membrane into the solvent by osmotic shock. It is known that some amino-acid binding proteins are readily liberated in this way, whereas others remain associated with the membrane. The glutamine binding protein evidently retains its binding activity in aqueous solution.

\title{
The Cell Coat and Transformation
}

ANY parameter of the cell surface which can be measured or assayed has attracted great attention recently, for it is clear that the transformation of nonmalignant cells to malignancy, either by tumour viruses or by chemical carcinogens, is accompanied by profound but subtle changes in the chemistry and architecture of the cell surface. One of the few parameters of the cell surface that can be measured by physical methods is the thickness of the cell coat. But as the ellipsometric measurements, reported by Mallucci et al. in Nature New Biology next Wednesday (February 16), indicate, any hope that a change in the thickness of the cell coat might prove to be a consistent feature of transformed cells must be forgotten.

As Mallucci et al. have found, mouse cells isolated from a tumour induced by polyoma virus have a coat at least twice as thick as that of untransformed mouse embryo fibroblasts but the coat of mouse cells isolated from tumours induced by methylcholanthrene is not significantly thicker than that of untransformed cells. Moreover, the rate at which virus transformed and chemically transformed cells resynthesize their coat after exposure to trypsin differs markedly, although in both cases it is faster than the rate in untransformed cells.

This resynthesis of coat glycoprotein is blocked to an equal extent in untransformed and both sorts of transformed cells by cycloheximide but the response of virus transformed and chemically transformed cells to actinomycin $D$ is very different. Although actinomycin D rapidly inhibits RNA synthesis and coat synthesis in untransformed and chemically transformed cells, the cells transformed by polyoma virus continue to make coat material for 100-220 minutes after exposure to the drug and after RNA synthesis has been more or less totally inhibited. It seems therefore that in the virus transformed cells, but not in the chemically transformed cells, the messenger RNAs with specific coat materials may have enhanced stability. These findings together indicate that as far as the thickness and synthesis of the cell coat are concerned, virus transformed and chemically transformed cells differ from each other at least as much as they differ from untransformed cells. 